\title{
APPROXIMATION OF BOUNDED VARIATION FUNCTIONS BY A BÉZIER VARIANT OF THE BLEIMANN, BUTZER, AND HAHN OPERATORS
}

\author{
VIJAY GUPTA AND OGÜN DOĞRU
}

Received 21 March 2005; Revised 13 May 2006; Accepted 28 May 2006

We give a sharp estimate on the rate of convergence for the Bézier variant of Bleimann, Butzer, and Hahn operators for functions of bounded variation. We consider the case when $\alpha \geq 1$ and our result improves the recently established results of Srivastava and Gupta (2005) and de la Cal and Gupta (2005).

Copyright (c) 2006 Hindawi Publishing Corporation. All rights reserved.

\section{Introduction}

Bleimann et al. [3] introduced an interesting sequence of positive linear operators defined on the space of real functions on the infinite interval $[0, \infty)$ by

$$
L_{n}(f, x)=\sum_{k=0}^{n} b_{n, k}(x) f\left(\frac{k}{n-k+1}\right), \quad x \in[0, \infty), n \in \mathbb{N},
$$

where

$$
b_{n, k}(x)=\left(\begin{array}{l}
n \\
k
\end{array}\right) \frac{x^{k}}{(1+x)^{n}}
$$

The Bézier variant of these operators for $\alpha \geq 1$ is defined in [6] as

$$
L_{n, \alpha}(f, x)=\sum_{k=0}^{n} Q_{n, k}^{(\alpha)}(x) f\left(\frac{k}{n-k+1}\right), \quad x \in[0, \infty), n \in \mathbb{N},
$$

where $Q_{n, k}^{(\alpha)}(x)=J_{n, k}^{\alpha}(x)-J_{n, k+1}^{\alpha}(x)$ and $J_{n, k}(x)=\sum_{j=k}^{n} b_{n, j}(x)$.

As a special case $\alpha=1, L_{n, \alpha}(f, x)$ reduce to the operators $L_{n, 1}(f, x) \equiv L_{n}(f, x)$, defined by (1.1). Some approximation properties of the Bleimann, Butzer, and Hahn operators were discussed in [1,2], and so forth. Very recently, de la Cal and Gupta [4] and Srivastava 
2 Approximation by a Bézier variant of the $\mathrm{BBH}$ operators

and Gupta [6] studied the rate of approximation for the Bleimann, Butzer, and Hahn operators and its Bézier variant $(\alpha \geq 1)$, respectively.

We recall the Lebesgue-Stieltjes integral representation

$$
L_{n, \alpha}(f, x)=\int_{0}^{\infty} f(t) d_{t}\left(K_{n, \alpha}(x, t)\right)
$$

where

$$
K_{n, \alpha}(x, t)= \begin{cases}\sum_{k \leq(n-k+1) t} Q_{n, k}^{(\alpha)}(x), & 0<t<\infty \\ 0, & t=0\end{cases}
$$

In this paper, we give a different and improved estimate on the rate of approximation for functions of bounded variation on the Bézier variant of Bleimann, Butzer, and Hahn operators.

\section{Auxiliary results}

In this section, we recall two lemmas, which are essential for our main theorem.

Lemma 2.1 [6, Lemma 3]. For all $x \in(0, \infty), \alpha \geq 1$, and $k \in \mathbb{N}$, there holds

$$
Q_{n, k}^{(\alpha)}(x) \leq \alpha b_{n, k}(x)<\frac{\alpha(1+x)}{\sqrt{2 e n x}}
$$

Lemma $2.2[5$, Lemma 3]. For $x \in(0, \infty)$,

$$
\left|\sum_{k /(n-k+1)>x} b_{n, k}(x)-\frac{1}{2}\right| \leq \frac{|1-x|}{6 \sqrt{2 \pi(n+1) x}}+O\left(n^{-3 / 2}\right) .
$$

\section{Rate of convergence}

Our main result is stated as follows.

THEOREM 3.1. Let $f$ be a function of bounded variation on every finite subinterval of $[0, \infty)$. Let $f(t)=O\left(t^{r}\right)$ for some $r \in \mathbb{N}$ as $t \rightarrow \infty$. Then for $x \in(0, \infty), \alpha \geq 1$, and for $n \rightarrow \infty$,

$$
\begin{aligned}
& \left|L_{n, \alpha}(f, x)-2^{-\alpha} f(x+)-\left(1-2^{-\alpha}\right) f(x-)\right| \\
& \leq \frac{9 \alpha(1+x)^{2}}{(n+2) x} \sum_{k=1}^{n} V_{x-x / \sqrt{k}}^{x+x / \sqrt{k}}\left(f_{x}\right)+\frac{\alpha|1-x|}{6 \sqrt{2 \pi(n+1) x}}|f(x+)-f(x-)| \\
& \quad+\frac{\alpha(1+x)}{\sqrt{2 e n x}} \varepsilon_{n}(x)|f(x)-f(x-)|+O\left(n^{-1}\right),
\end{aligned}
$$


where

$$
\begin{aligned}
& \varepsilon_{n}(x)= \begin{cases}1, & \text { if } \frac{x(n+1)}{1+x} \in \mathbb{N}, \\
0, & \text { otherwise, }\end{cases} \\
& f_{x}(t)= \begin{cases}f(t)-f(x-), & \text { if } 0 \leq t<x, \\
0, & \text { if } t=x, \\
f(t)-f(x+), & \text { if } x<t<\infty,\end{cases}
\end{aligned}
$$

and $V_{a}^{b}\left(f_{x}\right)$ is the total variation of $f_{x}$ on $[a, b]$.

Proof. We have

$$
\begin{aligned}
f(t)- & 2^{-\alpha} f(x+)-\left(1-2^{-\alpha}\right) f(x-) \\
= & f_{x}(t)+2^{-\alpha}(f(x+)-f(x-)) \operatorname{sign}^{(\alpha)}(t-x) \\
& \quad+\left(f(x)-2^{-\alpha} f(x+)-\left(1-2^{-\alpha}\right) f(x-)\right) \delta_{x}(t),
\end{aligned}
$$

where

$$
\operatorname{sign}^{(\alpha)}(t-x):=\left\{\begin{array}{ll}
2^{\alpha}-1, & \text { if } t>x, \\
0, & \text { if } t=x, \\
-1, & \text { if } t<x,
\end{array} \quad \delta_{x}(t)= \begin{cases}1, & \text { if } x=t \\
0, & \text { if } x \neq t\end{cases}\right.
$$

Therefore, we can write

$$
\begin{aligned}
\mid L_{n, \alpha}(f, x)-2^{-\alpha} f(x+)- & \left(1-2^{-\alpha}\right) f(x-) \mid \\
\leq\left|L_{n, \alpha}\left(f_{x}, x\right)\right|+ & \mid 2^{-\alpha}(f(x+)-f(x-)) L_{n, \alpha}\left(\operatorname{sign}^{(\alpha)}(t-x), x\right) \\
+ & {\left[f(x)-2^{-\alpha} f(x+)-\left(1-2^{-\alpha}\right) f(x-)\right] L_{n, \alpha}\left(\delta_{x}, x\right) \mid, }
\end{aligned}
$$

and our first estimates are

$$
\begin{aligned}
& L_{n, \alpha}\left(\operatorname{sign}^{(\alpha)}(t-x), x\right)=2^{\alpha} \sum_{k>(n-k+1) x} Q_{n, k}^{(\alpha)}(x)-1+\varepsilon_{n}(x) Q_{n, k^{\prime}}^{(\alpha)}(x) \\
&=2^{\alpha}\left(\sum_{k>(n-k+1) x} b_{n, k}(x)\right)^{\alpha}-1+\varepsilon_{n}(x) Q_{n, k^{\prime}}^{(\alpha)}(x), \\
& L_{n, \alpha}\left(\delta_{x}, x\right)=\varepsilon_{n}(x) Q_{n, k^{\prime}}^{(\alpha)}(x) .
\end{aligned}
$$


4 Approximation by a Bézier variant of the $\mathrm{BBH}$ operators

Then we have

$$
\begin{aligned}
G:= & \mid 2^{-\alpha}(f(x+)-f(x-)) L_{n, \alpha}\left(\operatorname{sign}^{(\alpha)}(t-x), x\right) \\
& +\left[f(x)-2^{-\alpha} f(x+)-\left(1-2^{-\alpha}\right) f(x-)\right] L_{n, \alpha}\left(\delta_{x}, x\right) \mid \\
= & \left|2^{-\alpha}(f(x+)-f(x-))\left[2^{\alpha} \sum_{k>(n-k+1) x} Q_{n, k}^{(\alpha)}(x)-1\right]+(f(x)-f(x-)) \varepsilon_{n}(x) Q_{n, k^{\prime}}^{(\alpha)}(x)\right| .
\end{aligned}
$$

Using the mean value theorem, we get

$$
\left|\sum_{k>(n-k+1) x} Q_{n, k}^{(\alpha)}(x)-2^{-\alpha}\right|=\alpha\left(\xi_{n, k}(x)\right)^{\alpha-1}\left|\sum_{k>(n-k+1) x} b_{n, k}(x)-2^{-1}\right|,
$$

where $\xi_{n, k}(x)$ lies between $2^{-1}$ and $\sum_{k>(n-k+1) x} b_{n, k}(x)$. Because of Lemma 2.2, it is easily seen that the intermediate point $\xi_{n, k}(x)$ is close to $2^{-1}$ for sufficiently large $n$. Then we can write $\xi_{n, k}(x)=(2+\varepsilon)^{-1}$ for each $\varepsilon>0$. Thus, we have

$$
\left(\xi_{n, k}(x)\right)^{\alpha-1}=(2+\varepsilon)^{1-\alpha} \leq 1
$$

for each $\alpha \geq 1$. By using (3.9) and Lemma 2.2 in (3.8), we obtain

$$
\left|\sum_{k>(n-k+1) x} Q_{n, k}^{(\alpha)}(x)-2^{-\alpha}\right| \leq \frac{\alpha|1-x|}{6 \sqrt{2 \pi(n+1) x}}+O\left(n^{-3 / 2}\right) .
$$

Hence, by using (3.10) in (3.7) and Lemma 2.1, we obtain

$$
G \leq \frac{\alpha|1-x|}{6 \sqrt{2 \pi(n+1) x}}|f(x+)-f(x-)|+\frac{\alpha(1+x)}{\sqrt{2 e n x}} \varepsilon_{n}(x)|f(x)-f(x-)|+O\left(n^{-3 / 2}\right) .
$$

On the other hand, to estimate $L_{n, \alpha}\left(f_{x}, x\right)$, we break the Lebesgue-Stieltjes integral into four parts as follows:

$$
L_{n, \alpha}\left(f_{x}, x\right)=\left(\int_{0}^{x-x / \sqrt{n}}+\int_{x-x / \sqrt{n}}^{x+x / \sqrt{n}}+\int_{x+x / \sqrt{n}}^{2 x}+\int_{2 x}^{\infty}\right) f_{x}(t) d_{t}\left(K_{n, \alpha}(x, t)\right)
$$

then, by proceeding along the lines of [6], we get

$$
\left|L_{n, \alpha}\left(f_{x}, x\right)\right| \leq \frac{9 \alpha(1+x)^{2}}{(n+2) x} \sum_{k=1}^{n} V_{x-x / \sqrt{k}}^{x+x / \sqrt{k}}\left(f_{x}\right)+O\left(n^{-1}\right) .
$$

Using (3.11) and (3.13) in (3.5), we get the desired result. This completes the proof of Theorem 3.1.

Notice that for the case $0<\alpha<1$, these results can be found in [5]. 


\section{Acknowledgment}

The authors are thankful to the referees for making valuable suggestions leading to the overall improvement of this paper.

\section{References}

[1] U. Abel and M. Ivan, Some identities for the operator of Bleimann, Butzer and Hahn involving divided differences, Calcolo 36 (1999), no. 3, 143-160.

[2] _ Best constant for a Bleimann-Butzer-Hahn moment estimation, East Journal on Approximations 6 (2000), no. 3, 349-355.

[3] G. Bleimann, P. L. Butzer, and L. Hahn, A Bernště̀n-type operator approximating continuous functions on the semi-axis, Indagationes Mathematicae 42 (1980), no. 3, 255-262.

[4] J. de la Cal and V. Gupta, On the approximation of locally bounded functions by operators of Bleimann, Butzer and Hahn, Journal of Inequalities in Pure and Applied Mathematics 6 (2005), no. $1,1-10$, article 4 .

[5] V. Gupta and A. Lupas, Rate of approximation for Bézier variant of Bleiman, Butzer and Hahn operators, General Mathematics 13 (2005), no. 1, 41-54.

[6] H. M. Srivastava and V. Gupta, Rate of convergence for the Bézier variant of the Bleimann-ButzerHahn operators, Applied Mathematics Letters 18 (2005), no. 8, 849-857.

Vijay Gupta: School of Applied Sciences, Netaji Subhas Institute of Technology, Sector-3, Dwarka, New Delhi 110045, India

E-mail address: vijay@nsit.ac.in

Ogün Doğru: Department of Mathematics, Faculty of Science, Ankara University, 06100 Tandogan, Ankara, Turkey

E-mail address: dogru@science.ankara.edu.tr 


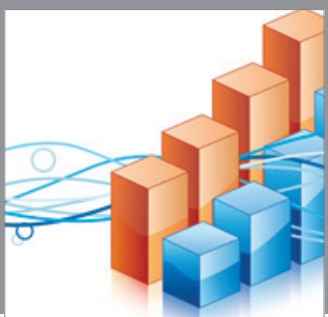

Advances in

Operations Research

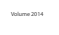

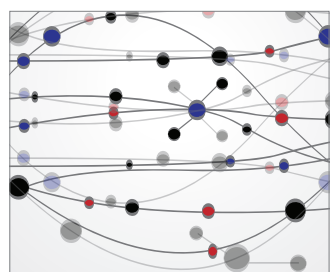

\section{The Scientific} World Journal
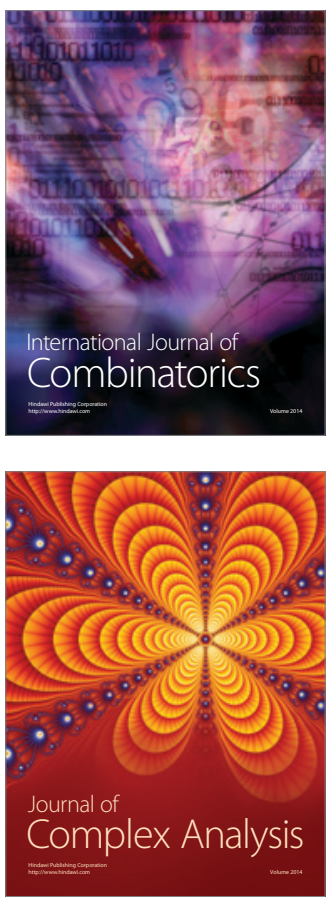

International Journal of

Mathematics and

Mathematical

Sciences
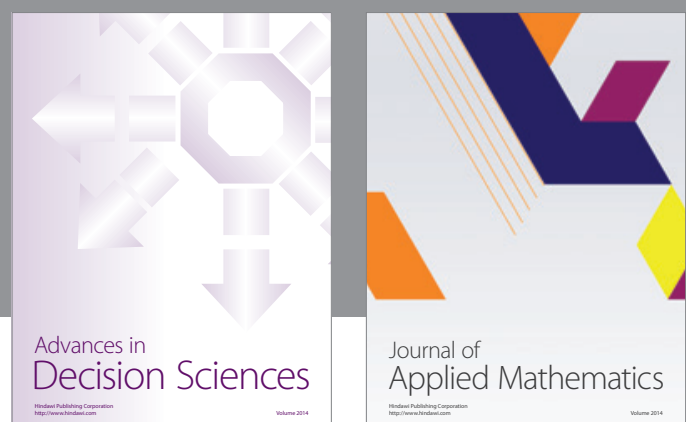

Journal of

Applied Mathematics
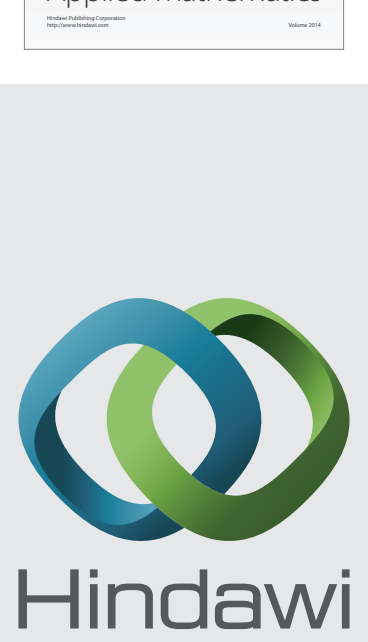

Submit your manuscripts at http://www.hindawi.com
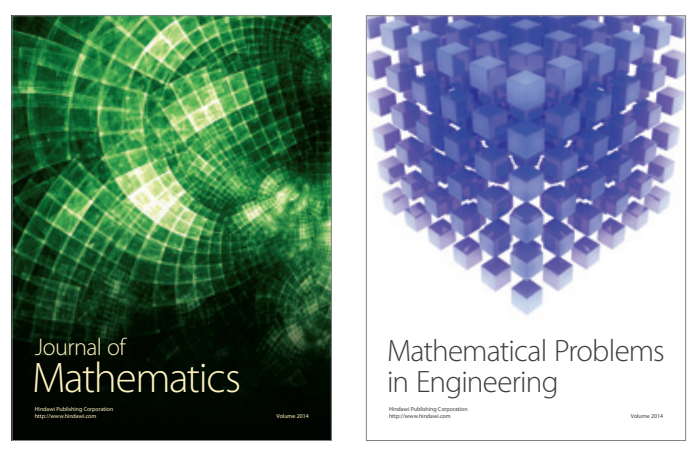

Mathematical Problems in Engineering
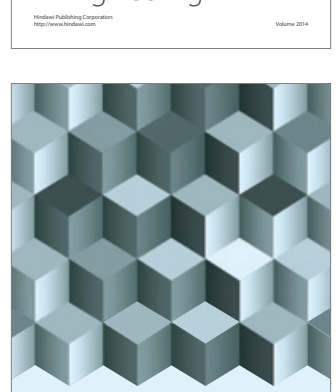

Journal of

Function Spaces
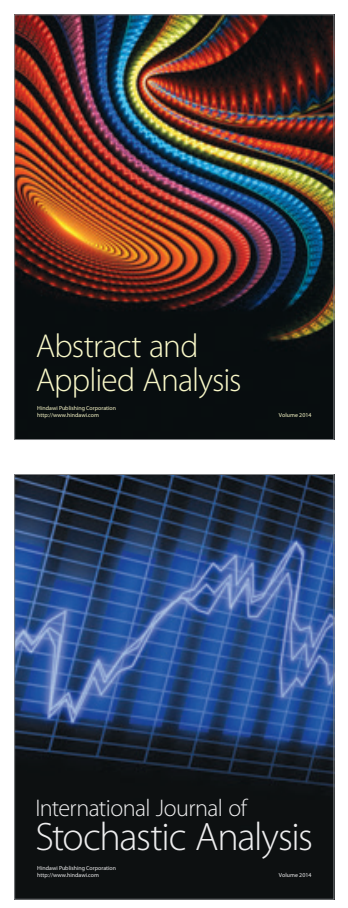

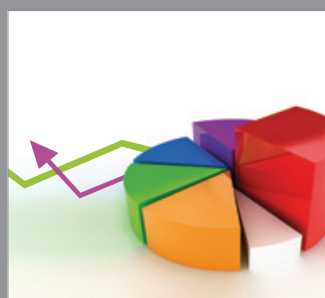

ournal of

Probability and Statistics

Promensencen
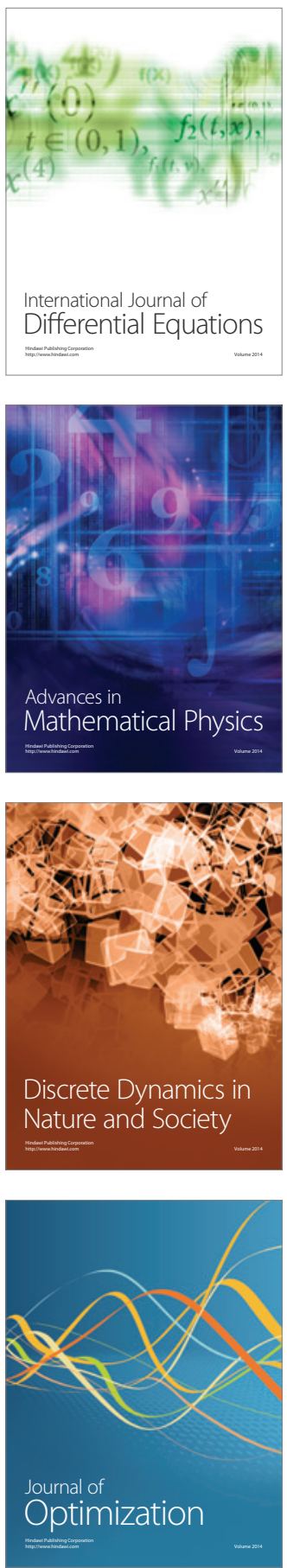